\title{
A DIGITAL DIAGNOSIS FOR THE «AUTUMN » STATUE (MARSEILLE, FRANCE) : PHOTOGRAMMETRY, DIGITAL CARTOGRAPHY AND CONSTRUCTION OF A THESAURUS
}

\author{
Roxane Roussel ${ }^{1}$, Marine Bagnéris ${ }^{2}$, Livio De Luca ${ }^{2}$, Philippe Bomblet ${ }^{3}$ \\ 1 1. Ecole nationale supérieure d'architecture de Marseille, 184 Avenue de Luminy, 13009 Marseille, France - \\ roxane.roussel@marseille.archi.fr \\ 2 UMR 3495 MAP CNRS/MCC, campus CNRS Joseph-Aiguier, bâtiment US, 31, rue Joseph-Aiguier, 13402 Marseille cedex 20, \\ France - marine.bagneris@marseille.archi.fr - livio.deluca@map.cnrs.fr \\ ${ }_{3}^{3}$ CICRP Belle-de-Mai, 21, rue Guibal, 13003 Marseille, France - Philippe.Bromblet@cicrp.fr
}

Commission II, WG II/8

KEY WORDS: Photogrammetry, point cloud, sculptural heritage, digital cartography, controlled vocabulary

\begin{abstract}
:
The evolution of the digital technologies allowed since the early 1990s, to multiply the photogrammetric acquisitions, the digital and mechanical diagnosis and the 3D diagnosis of the sculptural heritage, generating a better knowledge of these objects. The multiplication of these tools therefore allowed to establish multidisciplinary methodologies, generally leading towards less invasive restorations protocol. Here, the diagnosis of a case study is first accomplished, starting with a photogrammetric acquisition, then tested in the two and three dimension annotation platform named aïoli, in order to determine the current possibilities for an innovative use of these tools, benefiting at the diagnosis of the sculptural heritage. On the order hand, along with the multiplication of the multidisciplinary methodologies, a very contemporary problematic arises: the need to improve the communication and discussion between different professionals. The goal was here to offer innovative methods to give all professionals the same vocabulary, a shared knowledge, therefore allowing a better diagnosis of these pieces of art.
\end{abstract}

\section{INTRODUCTION}

A few decades ago, digital technologies began to take an increasingly important place in our lifestyle. Indeed, in both our daily lives and work, they gradually multiplied, and became essential in certain fields. For instance, computing is now necessary to conceive architecture : the use of computer assisted drawing and $3 \mathrm{D}$ modeling now appear to be standard.

In fields such as archaeology or in the analysis of artistic and architectural elements, different tools appeared. In the field of the statuary, photogrammetric acquisition is the starting point of a digitization. Thanks to precise photography techniques, it allows to reconstruct a point cloud on software such as Micmac or Photoscan. Some tools, such as Zbrush or 3ds Max, also make it possible to create skeletons, generated because of these acquisitions.

This study questions how these digital tools could be used during the diagnosis of statues, in order to turn them into interactive, evolutionary and accessible assets to the field and each one of its member.

To this end, it was first necessary to understand and measure their current use. Then, in order to grasp the possibilities, the limits and the evolutions that the digital tool offers today, it will be approached through a case study: the statue «the autumn» of the Magalone Gardens in Marseille. With it, an experimental diagnosis is meant to be created, using existing or current development platforms and software. The purpose of this first study is to evaluate the current possibilities, as well as the limits and the obstacles to the development of these solutions nowadays ; in order to draw conclusions about the contribution of these tools in the field of the diagnosis of statues.

Secondly, it was necessary to wonder how it could be possible to make these tools even more efficient and useful to all the professionals involved in the conservation and restoration process. The case study is then considered from a new angle: the shared vocabulary. It is then necessary to formulate an introduction to the concept of ontology, in order to mesure if the study of the vocabulary and the introduction of the domain ontology can allow us to collect more data on this piece of art.

The purpose of this experimental work is consequently to measure whether these approaches, very different from the traditional expertise, make it possible to question the current mechanisms of knowledge production.

\section{THE DIAGNOSIS TODAY AND ITS PERSPECTIVES}

In the South of France, the diagnosis and restoration of the sculptural heritage began using the potentialities of a 3D model in 1994 and 1998, with the analysis of the statue Auguste and the statue Neptune (Coignard et al., 2009) both preserved in the city of Arles. At the time, the lack of funds and time, added to the fact that digital technologies were still quite unknown, were obstacles to the fulfillment of these practices. However, in 1998, with the Neptune statue (Figure 1), an antique of great quality, this method was again applied, and questioned, therefore, pursuing this innovative approach. Indeed, it yet had little opportunity to be implemented, despite its 20 years of existence.

In this work, the team also mentioned the possibility to use laser scanning on an existing model. It would to provide assumptions 
about the original position of missing limbs through a comparison of the statue's muscle expression. On the other hand, an integration of the statue's structure in its 3D model was considered, in order to submit it to static, seismic or thermal analysis, allowing to predict the evolution of the alterations over time. The communication and description of the mechanic efforts in the global structure should, by extension, be facilitated, allowing the damages of the statue to be prevented. Finally, this technology would offer new solutions for the presentation and exhibition of the art piece and its foundation. It is, therefore, interesting to see that less than 6 years later, in order to straighten and base an other piece of art, the statue of the emperor, now located in Alba la Romaine, this work is finally realized.

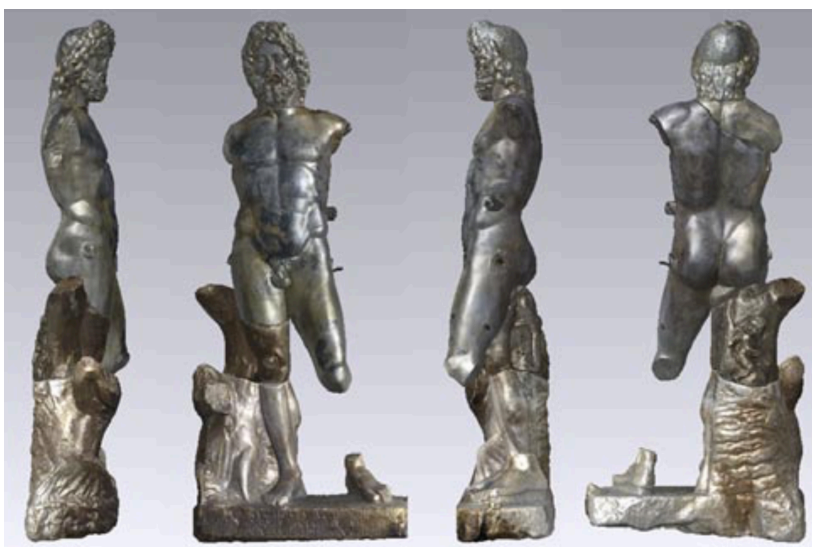

Figure 1. Picture of the Neptune statue (Coignard et al., 2009)

The evolution of the digital tools allowed, during the last decade, to multiply the photogrammetric acquisitions, the digital and mechanical diagnosis and the 3D diagnosis, generating a better knowledge of these objects. The application to the imperial marble statue of Alba-la-Romaine (Bagnéris et al., 2014) is a successful example of these multidisciplinary methodologies, authorizing a better understanding of the mechanical behavior and the history of the piece of art. Indeed, the work began with a photogrammetric acquisition allowing to generate a $3 \mathrm{D}$ model, providing a valuable insight for the physical characterization and numerical analysis. Moreover, the ultrasound analysis and the use of FEM/DEM analysis permitted to create a non-invasive and reversible pedestal solution (Figure 2).

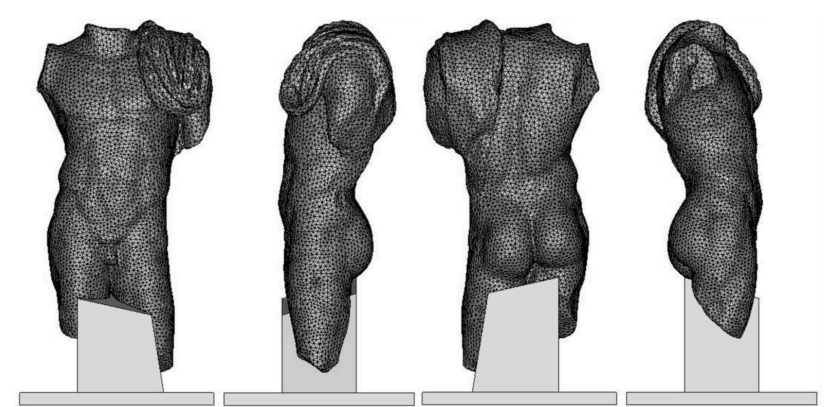

Figure 2. Picture of the project for the base of the statue (Bagnéris et al., 2014)

Thereby, the field of the sculptural restoration seems to create more respectful and discreet methods, by seeking innovative ways to generate new knowledge on this sculptural heritage.
This example therefore is an application of the aspirations raised in projects such as Neptune. Here, the tool has accompanied an evolution of mores and practices in the field, which gradually becomes less invasive (Borri, A., et Grazini, A., 2006). Finally, these studies seem to consider themselves as stages in the history of the piece of art, allowing us to believe that in a few decades, it will be possible to bring new knowledge on these works (Michel, L., 2013).

\section{THE CASE STUDY : THE MAGALONE STATUES}

This work can be considered an experimental setting, aiming to create a digital diagnosis for a case study, to see the current possibilities, and the next goals. This experience is focused on the use of photogrammetric techniques and the creation of digital cartography, to reveal the benefits of digital monitoring, and show that the current tools already enables users to reveal new data. The work was initiated in Marseille's national architecture school, with the collaboration of the UMR 3495 MAP, building together a methodology for the valorization of the sculptural heritage.

\subsection{The Autumn Statue}

The case study is the statue "Autumn », located in Magalone garden, in Marseille (France). This piece of art, of which the sculptor is still unknown, is one of the garden four statues, each representing a season. Its creation dates back to the end of the XVIIth century. Two of them represent feminine figures (spring and summer), while the two others are masculine figures (autumn and winter). They all are placed around the central floor, along the aisles. Spring and autumn are placed on the south side, summer and winter on the north side.

The Magalone gardens are located in the 8th district of Marseille and its residential neighborhood. They stand along the Boulevard Michelet, one of the main avenues crossing the city. The garden is also in front of the building " la cité radieuse ", built by Le Corbusier between 1947 and 1952, now classified as a historical monument, and on which one has a direct view from the gardens, especially in winter, when trees loose their leaves. Nowadays, its conservation is handled by the CICRP (Heritage's conservation and restoration multidisciplinary center).

In 1999, the city's Ecology and Green Spaces Department decided to proceed with a restoration. The CICRP then helped the city analyze the offers. The Mérindol workshop completed the work the same year, according to an intervention protocol defined by the CICRP and the conservators in charge (Figure 3 ). The alterations of the statues at the time and the restorations executed will be discussed through the case study, mentioned in the next section.
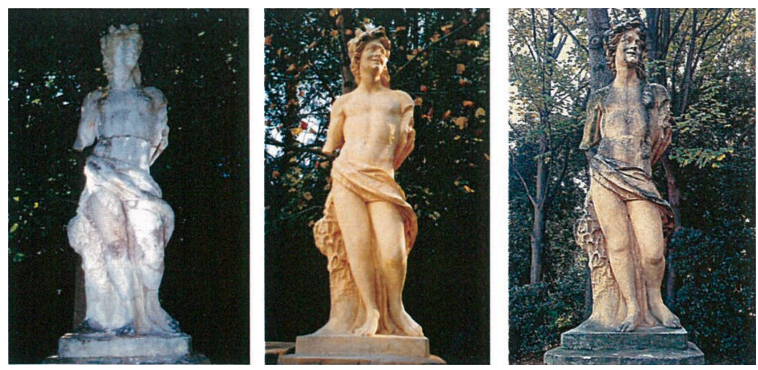

Figure 3. Photographies of the statue un 1999 before restorations, after restorations, and in 2011 (Vallet, J.M., et Mérindol, P., CICRP, 2011) 
In 2011 a new diagnosis was requested and executed to measure the method's efficiency and the current state of conservation of the statues. The report of the CICRP «Impact of the restoration of four statues of the garden of Magalone (Marseille, France) after twelve years of in situ conservation» (Vallet, Mérindol 2011) analyses the choices made and the methods used in 1999 during the restoration process. It provides a critical feedback to measure the effectiveness of these methods, the current state of preservation of the statues, and whether protections are still effective a decade later. The alterations are then precisely listed and compared with those noted in 1999 (Figure 2).

In April 2018, a management plan for the gardens was developed (Bromblet, 2018),(Jardins d'histoire, sce ateliers up+, 2018). It then looks back on the history of the building, establishing a landscape analysis added to an analysis of the management of the water, the decorative elements, the sculptural elements (greenhouse, rock, benches, stairs), the accesses, the limits and uses of the garden.

\subsection{Acquisition and point cloud processing}

The work here started with the statue's photogrammetic acquisition, according to a protocol previously established. The acquisition of a statue, because of its details and the necessity to have a $360^{\circ}$ view of the object, required a large number of photographs to be taken. It was necessary to photograph three turns of the statue, one from the bottom, looking at the statue, one facing the statue, and a last one seen from above. The point cloud was then transformed into a point cloud generated with the mic-mac open source of tools. The digital cartography was then generated on the aïoli platform (De Luca, L., 2018). This platform wishes to bring together the different actors involved in documenting and sharing the cultural heritage through new practices, therefore allowing a better understanding of these objects (MAP-GAMSAU Development Team, 2019). In 2016, the thesis of Adeline Manuel, defended at the National School of Arts and Crafts of Paris, marked the beginning of this work (Manuel, 2016). In the latter, built out under the direction of Livio de Luca, they discuss the fact that semantic annotations on 3D models are still insufficient to effectively describe the heritage artifacts.

Each user is here allowed to annotate the object, leading to create a bridge between the object and the information produced by a community. Moreover, the platform enables a multitemporal analysis, allowing a follow-up of the state of its conservation. It is currently developed in the MAP laboratory (models and simulations for architecture and culture heritage) with the help of the CNRS (national center for scientific research) and the French ministry of culture. About 70 photos were used to reconstruct the cloud, and build the cartography. The cloud is dense enough to allow a comprehension of the statue and its form. Since the statue is very eroded, it no longer shows a lot of details in drapes, hair or facial features. This allows to work with a less dense cloud, without losing important information about the reliefs of the statue. However, we can admit there is a lack of information at the top of the skull.

\subsection{Digital cartography}

With the help of aïoli and photoshop, the digital cartographies were then created. They allowed, with the help of the former follow-up, to create a clearer version of the expertise, by showing the location of the components, the alterations and the 1999 restoration. These cartographies were based on a photography taken for the photogrammetric acquisition, which was used to create the cartography of the materials. All cartographies were created on the same photography, then allowing to compare them to each other. On the other hand, this simulation does not allow, contrary to what will then be allowed by aïoli, to see all sides of the statue, and thus its annotations in three dimensions.

They were all created by confronting the ancient expertise with photos taken in the corresponding years. As a consequence, when an alteration or a material was mentioned in the followup, its specific location on the statue was then researched with the help of these pictures. Different mapping of the statue's alterations were created, one for the year 1999 before the restoration, one for 2011 and one for 2018. They were each divided into seven categories including: cracks, detachment, material loss, discoloration and deposit, biological colonization and finally, former restorations. The categories were identical for each period, to allow a themed comparison and not only a comparison through time. Indeed, with the platform each one could be seen without the others, but in the three periods of time.

Having only a photograph of the condition of the statue in 1999 before the renovations, it was sometimes difficult to identify the location of the alterations. On the base for example, no alteration were placed since the photo did not allow to observe its condition. This cartography (Figure 4) highlights the importance of the grey coloring on the statue, which covers its upper half. On the lower part, the scaling of the stone are quite noticeable, and give an uneven appearance to the models of the legs. The development of lichens are the last major alterations observed.

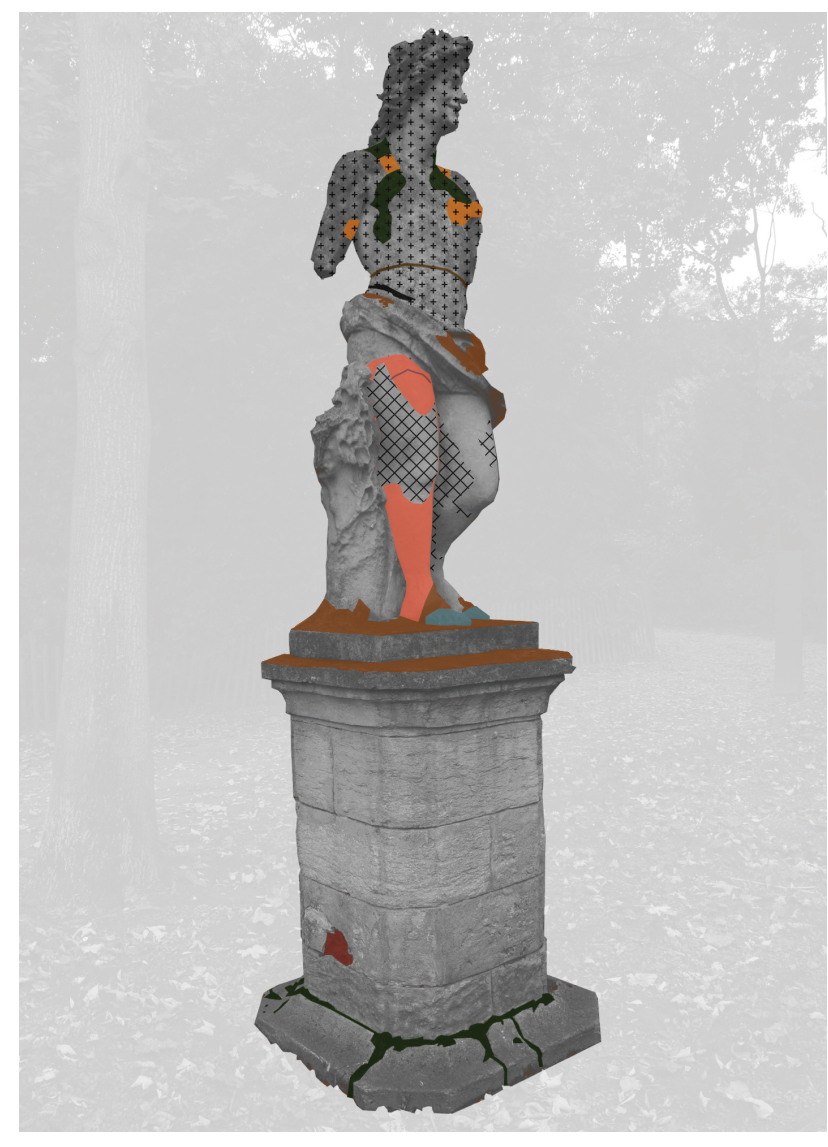

Figure 4. Alterations in 1999 
The alterations observed in 2011 are different from those observed before any contemporary renovation. Indeed, the protective layer applied still covers the statue almost completely, although some areas of shelling are noticeable (Figure 5). On the other hand, biological colonisations are largely present on the statue, mainly because of the methods of watering used in the grass areas, which sometimes come to water the bottom of statue. On the other hand, the chromatic alterations are very noticeable : the base adopts a grey coloration while the bust of the statue bleached because of the leaching of the stone. Finally, fossils are exposed because of the bleaching. It is noted that the deterioration in 2011 and 2018 are much similar to each other, compared to those observed in 1999. This seems logical since no restoration of the statue was completed during this second period of time.

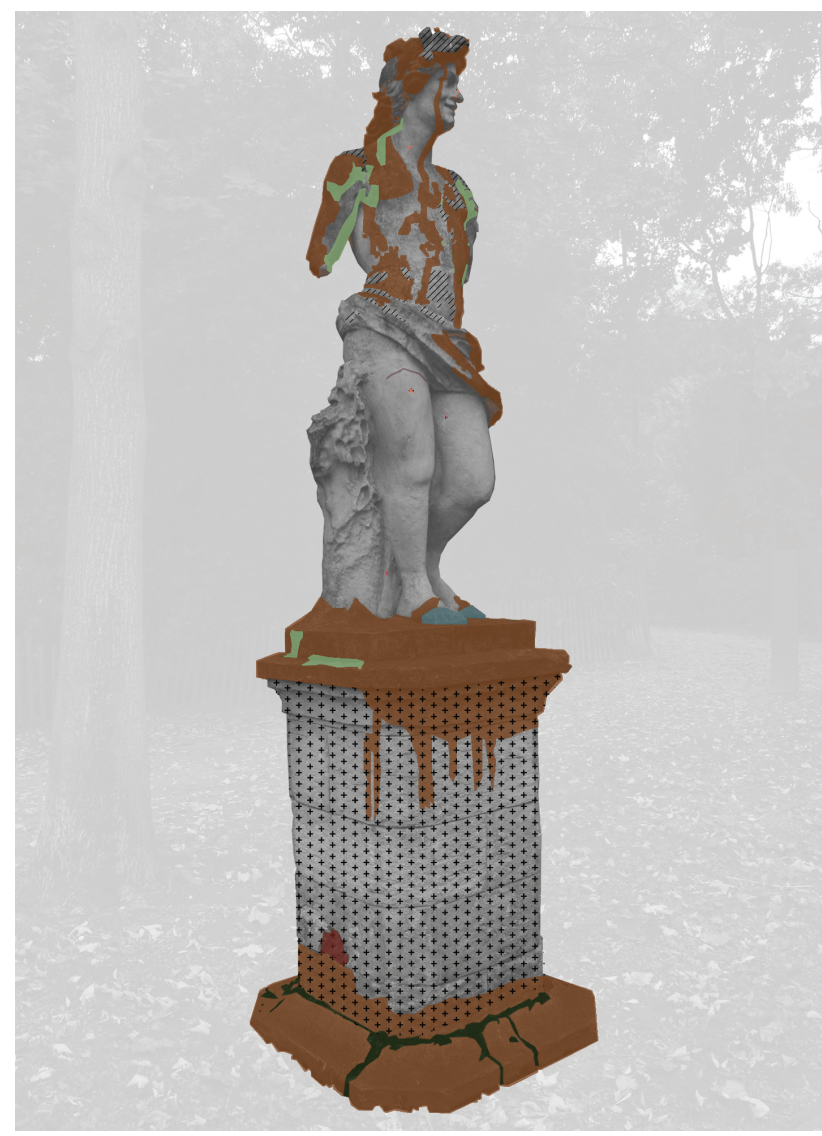

Figure 5. Alterations in 2011

Nevertheless, this confirms the effectiveness and durability of the protective layer, which, almost 20 years after its application, still makes it possible to mask old restorations. On this statue, the layer of protection does not seem to have tarnished between 2011 and 2018.

However, the alterations already observed in 2011 are still noticeable and even extended (Figure 6). While bleaching and biological colonization constantly develop, there is always an important grey coloring on the base. Colonizations seem to have gained importance on the skull of the statue, gradually covering the whitening of the stone. They also take up more and more space on the base.

The nature of colonization sometimes changes. Thus, in most areas, the location of biological colonization has not changed, but progressively become more green than black.
Consequently, these digital cartographies allow the collection of multiple data, as well as identifying and inventorying the different states the sculptural heritage has gone through. In the future, similar cartographies could be created to try to understand the evolution and components of the statue. Cartographies could be established over the years, each expert producing a map joining their report, and allowing a time lasting monitoring.

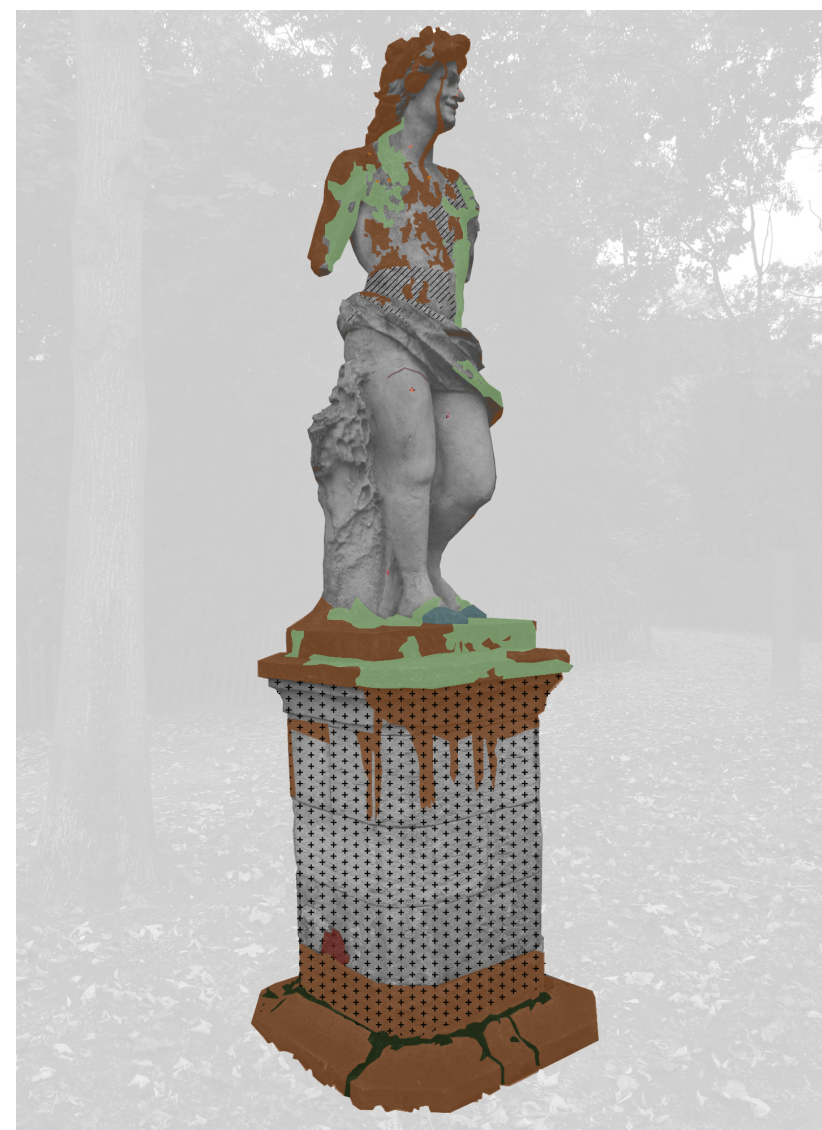

Discoloration \& deposit

Cracks

锤热 Grey colouration Ancient crack

WVI/, Bleaching

Detachment

disintegration of the protective layer Stone scaling

Black alga

Orange lichen

Material loss Green lichen

\section{Erosion}

Loss of components

Black crust

Joint 


\subsection{A new approach for the diagnosis}

This map (Figure 7) shows the evolution of alga's location through the years. It here shows a different approach than in a traditional report. Indeed, the previous maps showed the content of a traditional follow-up, but with a visual medium instead of a written one. Here, we can see an innovative use for the diagnosis of sculptural heritage. Indeed, we could imagine that each expert could create his own spatial annotations: through the years and thanks to the many maps created, it would then be possible to observe a specific type of alteration, and its evolution. Consequently, they allow to quantify and localize the alterations.

Despite the fact that these software are not yet usable, it already appears that these tools allow a huge step towards multiplication and democratization of these digital diagnosis. Allowing a better visualization than a text, it seems to generate a better understanding of the case study, and, consequently, of the sculptural heritage. This time saving can even be noticeable before the elaboration of a diagnosis: users can $\log$ in the platform, analyze what has already been done, and compare it directly to their own observations.

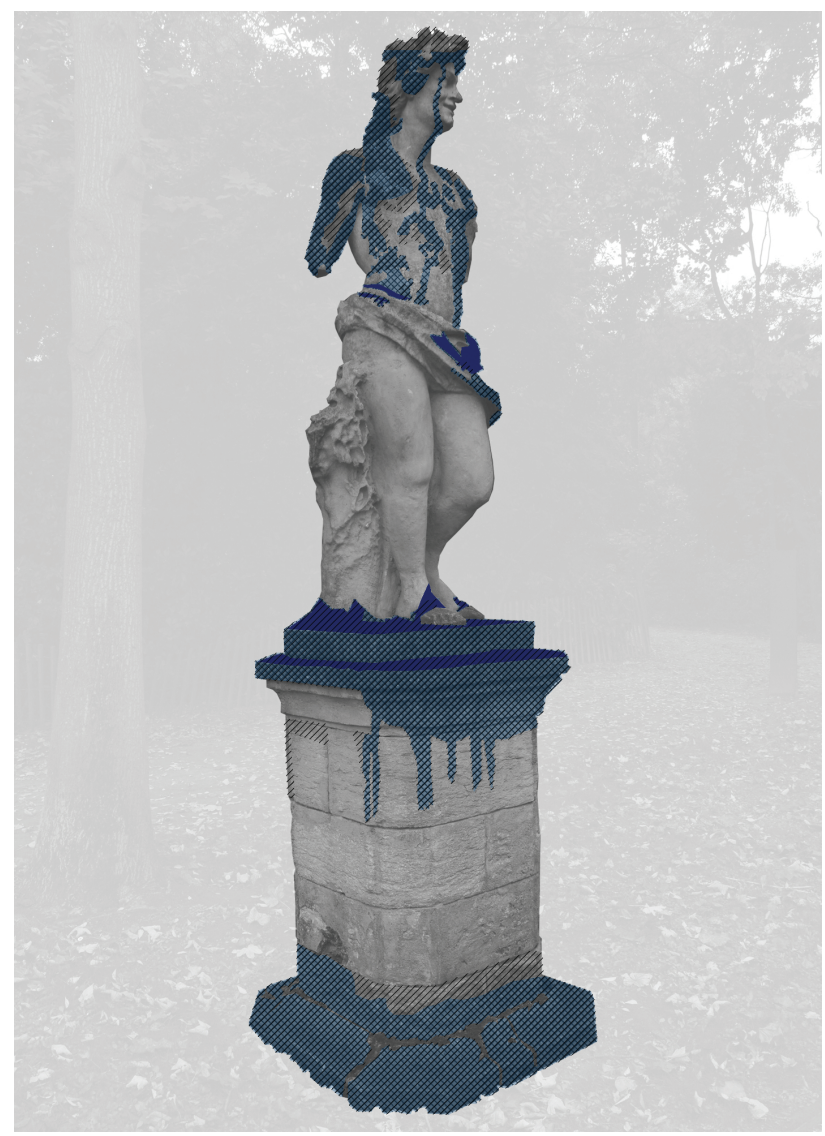

Figure 7. Evolution of Alga between 1999 and 2018

\section{DOMAIN VOCABULARY}

\subsection{The need for a shared vocabulary}

With the development of this multidisciplinary approach in the field of diagnosis and renovation, a very contemporary problematic arises: communication and discussion between the different professionals. Indeed, conservators, scientists and engineers now have to communicate daily.
Yet, their different paths and education can lead to misunderstanding, due to the use of different vocabulary. Moreover, these professionals are then led to exchange with the architects responsible for the conservation of these objects. The same difficulties are then noted.

Indeed, depending on the field, the vocabulary used may be different, and a word can have different meanings or a slightly different definition. While the collaborations multiply and normalize, it seems legitimate to imagine a common language, in order to avoid misunderstandings and to allow greater efficiency of work. This desire to create a consensus of terms, a universality of their definition is a goal often mentioned in the preface of glossaries or vocabularies published online.

By extension, there is a great challenge before us : the diffusion of the vocabulary, the accessibility of a precise, scientific and cultural knowledge. This goal would allow to give all professionals the same tools, the same knowledge, therefore allowing a better diagnosis.

This analysis of the "Autumn » statue enabled to pursue the digital and centralized diagnosis through another perspective : the creation of a domain vocabulary. Indeed, with the multiplication of multidisciplinary teams, the need to improve the communication between the different professional bodies appeared. Creating a shared vocabulary has the benefit of offering each expert the same tools, and assuring a better understanding of every teammate. Consequently, each word has a specific meaning, understandable by every member of the team.

With the correlation of visual data being experimented, the need to define conceptual scaffolds, related to a domain knowledge formalization, allowing to select an appropriate vocabulary for each element, remained.

\subsection{Towards a centralized platform for the diagnosis of the sculptural heritage}

Aware of the challenge represented by the opportunity to develop a common vocabulary, more and more writings work on an innovative proposition allowing to enjoy the knowledge of the actors and experts involved, and the many terms and vocabulary used by the various professionals : scientists, craftsmen, conservation professionals... Several ideas emerged. First, the idea of creating a centralized platform allowing to centralize the diagnosis of sculptural objects.

This work focuses on the interest of proposing a possible structure for the annotation in two and three dimensional semantic annotation platforms, in order to integrate a defined vocabulary. Each of the actors would thus have access to the same terms, as well as to the same structuring of these terms, thus offering everyone the same tools to communicate the knowledge they collected.

In addition, this would imply great opportunities for crosschecking data, between different period of time (comparing current annotations with older ones) and between different experts (it would then be possible to display layers, some information and then compare them to each other).

However, before beginning this work and considering any form of implementation within a digital tool, we need to understand the necessary steps to structure the annotations within such tools. 


\subsection{Towards a domain Ontology}

The word « ontology » is now used in the digital field to describe a structured set of concepts and terms, representing the meaning of a field of information. Borst explained in 1997 that the ontology seeks to create an explicit and formal specification of shared conceptualization. Thus, ontology creates a semantic structuring (in the same way a thesaurus does) and a formal structuring (Messaoudi et al., 2018). In other words, the concepts and link between them must be explicitly defined, but the ontology must also be understandable by a computer.

It necessarily relies on a controlled vocabulary as well as on clearly defined concepts, and is composed of classes and properties that allow to specify the rules of inference. The properties connecting these concepts can belong to two categories: data properties and object properties. Data properties are used to describe a relationship between an element and a data value. Object properties describe the relationships between the elements, and can be functional, symmetrical, reflective, etc.

\subsection{Documentation phase}

A documentation and decision making phase first started. The first task, introduced during the establishment of the map's legends, was to define the vocabulary used to nominate the statue, its alterations, components and surface. The subsumption links between all of them were not yet established at that point. With this selection of terms came their definition. During this experience, many arose from the " illustrated glossary on stone deterioration patterns " created by the ICOMOS (Anson Cartwright, T., Vergès-Belmin, V., 2008)(Grimmer, A.E., 1984), this source having already been the matter of discussions and of a scientific consensus. To understand this list and its division into several categories, it is important to note that the choice and definition of words took place in conjunction with the class definition. Definitions must then be collected and established in collaboration with professionals of the field.

\subsection{Structuration phase}

It was then necessary to highlight the fields of knowledge corresponding to the classes of description of the conservation of the statuary heritage. These classes will then become the heart of domain ontology. The documentation phase, through the study of terms, as well as of various publications, vocabularies, and reports, have helped to highlight the various aspects of the study of such a piece of art, and the concepts necessary for their description. The categories were finally established, every one being sufficient and necessary, creating the heart of the ontology : Statue, theme, production technique, components, alterations. Each word was then turned into a concept or a class.

\subsection{The taxonomic representation}

Then, the semantic structure transformation into a taxonomic representation was needed. Each concept was then hierarchically linked to another.

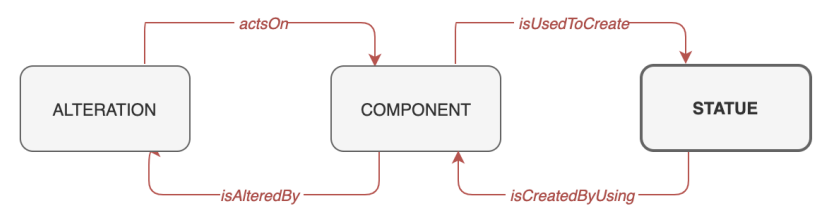

The first proposition (Figure 8) is representing how the vocabulary had been considered during the creation of the cartographies.

However, the notions of thematic and production techniques had to be added, in order to also characterize the form of the statue (Figure 9). What it represents both figuratively and metaphorically is represented by the «thematic» class, while its construction is explained within the «production technique» class.

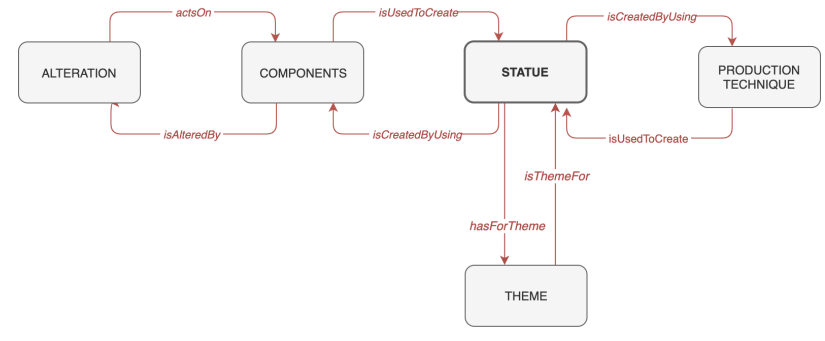

Figure 9. Second proposition

Moreover, all the interventions on the form of the work can be considered as production techniques, since the addition of all these interventions, over the years, have contributed to give it its current form and appearance.

The construction of the work as well as the actions completed by the professionals of the restoration can thus all be included in the class of production techniques (Figure 10). On the other hand, if some previous actions have damaged the work, they will be found in the alterations.

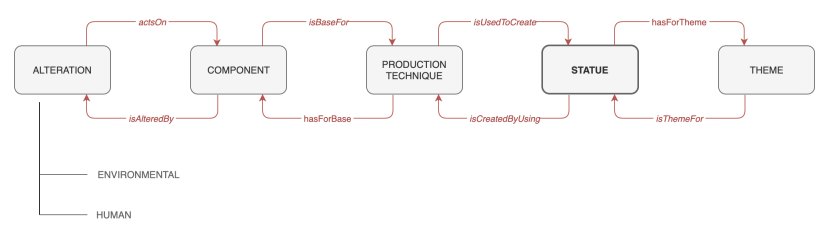

Figure 10. Final proposition

The work of the experts, who are at the service of the sculptural heritage, is distinct from the human actions that could have damaged it.

Finally, it seems necessary to differentiate human and environmental alterations within the " alteration » class in order to separate the harmful action (whether they are direct or not), from those related to the unfavorable environment in which the piece of art is located. This leads to the following modeling.

\subsection{The subclasses}

After the definition of the ontological, the subclasses were defined. Each of the subclasses can have up to five levels of concept.

They correspond to the terms defined and listed during the first phase, known as the documentation phase. Here is an example of one class : alteration (Figure 11) (Figure 12).

Figure 8. First proposition 


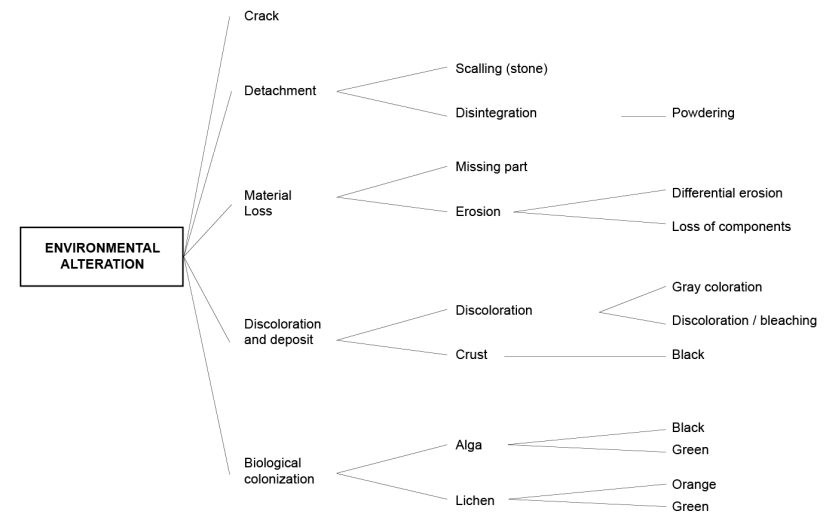

Figure 11. Environmental alterations

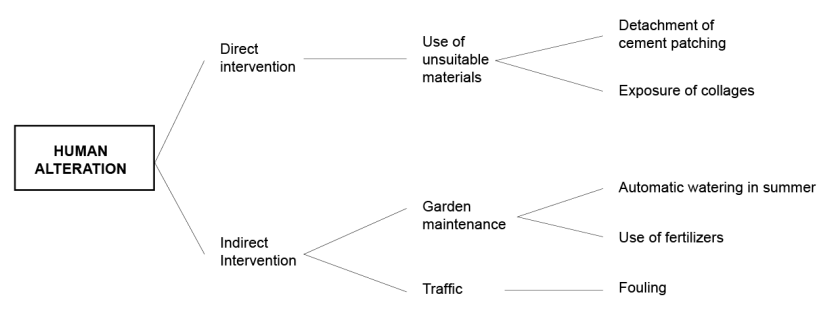

Figure 12. Human alterations

\section{THE FUTURE OF THIS WORK}

\subsection{Expand this experimental work to the entire field}

The next step, to transform this work into a domain ontology is to extend the work done for a single case, to the whole field of the conservation of the sculptural heritage. In fact, it would be necessary to extend the different levels of concepts and enrich them by adding all the terms necessary to describe and qualify any object.

\subsection{A merge with CIDOC CRM}

Once this work completed and extended to the entire field, the ontology core then defined will be formalized with a protected ontology publisher.

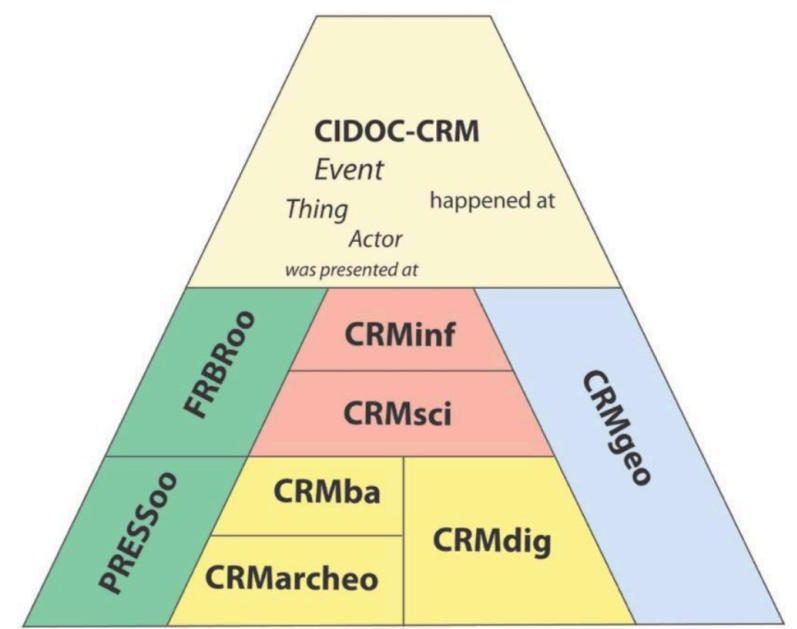

Figure 13 : Presentation of the CIDOC-CRM
It would then be relevant to consider a merger with CIDOCCRM (Figure 13), a reference conceptual model and a generic ontology of reference, in the field of scientific documentation of cultural heritage. Once completed, a correlation, thanks to SQL/ SPQRL mapping, can then be created between the databases within the information system.

\subsection{An implementation within the aïoli platform to create new data}

The work previously created with the semantic annotation platform aiioli, also encourages to envision the implementation of this ontology within the platform. At the moment, there is no possible structure for the annotations in the platform, each name being entered manually.

Nevertheless, the creation of an annotation system organized with an ontology would have several advantages. First of all, it would allow an efficient structure of the different annotation layers, classifying and organizing them according to the defined model. In addition, it could allow to cross the information.

\section{CONCLUSION}

This data organization, associated with the digital analysis created, now allows to envision the extraction of new information and knowledge because of innovative digital tools and platforms. Indeed, this analysis seems to show the benefit of creating visual cartography in addition to the traditional follow-up, therefore allowing a better understanding of the different states the statue went through, as well as a direct comparison of them.

Moreover, the definition of a domain vocabulary points out the importance of offering shared tools to every member of the multidisciplinary teams, allowing a better knowledge and communication for everyone.

As a consequence, this experimental work on a limited scale allowed to test the principles that could later be extended to an entire corpus. They would then allow a crossed comparison and the establishment of conclusions about a correlation between environmental conditions and alterations.

\section{REFERENCES}

Anson Cartwright, T., Vergès-Belmin, V., 2008. ICOMOS and International Scientific Comittee for Stone, Illustrated Glossary on Stone Deterioration.

Bagnéris, M., et al., 2017. A complete methodology for the mechanical diagnosis of statue provided by innovative uses of 3D model. Application to the imperial marble statue of Alba-laRomaine (France). In: Journal of Cultural Heritage, http:// dx.doi.org/10.1016/j.culher.2017.05.002.

Borri, A., et Grazini, A., 2006. Diagnostic Analysis of the Lesions and Stability of Michelangelo's David. In: Journal of Cultural Heritage 7, no 4, https://doiorg/10.1016/j.culher. 2006.06.004.

Bromblet, P., 2018. Etude de l'état de conservation de la statuaire et de son évolution depuis 2011, préconisations générales. Marseille, France.

Coignard, B., Coignard, S., 2009. Neptune, étude préalable par simulation informatique et restauration. Musée départemental de l'Arles antique, Rapport d'intervention, Dreux. 
De Luca, L., 2018. Aïoli, une plateforme d'annotation sémantique 3D pour la documentation collaborative d'objets patrimoniaux. In: Culture et recherche $\mathrm{n}^{\circ} 137$ (spring/summer 2018), pp. 41-42.

Grimmer, A.E., Department of the interior national park service preservation assistance division, 1984. A glossary of historic masonery deterioration problems and preservation treatments.

Jardins d'histoire, sce ateliers up+, 2018. Jardin de la Magalone Elaboration d'un plan de gestion, Phase 2, diagnostic de l'état actuel, Marseille, France.

Manuel, A., 2016. Annotation sémantique 2D/3D d'images spatialisées pour la documentation et l'analyse d'objets patrimoniaux. Thèse de doctorat en Science des Métiers de l'ingénieur, sous la dir. de De Luca, L,. Paris, École Nationale Supérieure d'Arts et Métiers.

Messaoudi, T., Véron, P., Halin, G., De Luca, L., 2018. An ontological model for the reality- based 3D annotation of heritage building conservation state. In: Journal of Cultural Heritage, Elsevier, 29, pp.100-112. 10.1016/j.culher. 2017.05.017. hal- 01982887.

Michel, L., 2013. Méthodologie d'analyse structurelle et de restauration d'œuvres sculptées. Thèse, université d'Orléans.

Riccardelli, C., Morris, M., Wheeler, G., Soultanian, J., Becker, L., et Street, R., 2014. The Treatment of Tullio Lombardo's Adam : A New Approach to the Conservation of Monumental Marble Sculpture. In: Metropolitan Museum Journal 49: 48-116. https://doi.org/10.1086/680027.

Vallet, J.M., et Mérindol, P., CICRP, 2011. Impact de la restauration de quatre statues du jardin de la Magalone (Marseille, France) après douze années de conservation in situ. 